\title{
RFID Based Centralized Patient Monitoring System and Tracking (RPMST)
}

\author{
Dr. S. Padmapriya M.E, PhD (Guide), ${ }^{1}$ Indu Goel, ${ }^{2}$ A.Sunitha, ${ }^{3}$ P.Arul \\ Head of the Department (IT) \\ Prathyusha Institute of Technology and Management \\ 1, 2, 3(Information Technology, Prathyusha Institute of Technology and Management/Anna University, India)
}

\begin{abstract}
Radio Frequency Identification (RFID) though finds its use in many applications, still has to be accepted in a clear view. Talking in the perspective of a patient the only thing that strikes first is that lots of treatments undergone, many health records to be maintained, and after which remembering the concerned doctor's name and other details. This becomes impossible at a certain point of time when the details have to be conveyed. To make it easier this concept of maintaining a centralized information system and sharing has been proposed through the use of RFID technology. RFID is known for its unique ID number. Using this advantage, in the first section the monitoring of a patient is done whenever he arrives at the hospital. In case of any shifts from the hospital the information is still available. Every patient is provided with a unique RFID number and all the details regarding the patient and treatments are stored in a centralized database which is retrieved by the server. The second section is patient tracking where in case of any emergency in a closed environment the patient is provided with assistance in a short span of time. The patient is regularly monitored by the temperature and heartbeat sensor. The moment the value crosses the normal range a message is sent through GSM/GPS to the nearest hospital with its location and also to a relative. Assistance is provided accordingly to the patient.
\end{abstract}

Keywords: GSM Modem, MAX 232 IC, PIC micro-controller, RFID Reader, RFID Tag.

\section{INTRODUCTION}

RFID stands for Radio Frequency Identification. RFID is one member in the family of Automatic Identification and Data Capture (AIDC) technologies and is a fast and reliable means of identifying objects. There are two main components: The Interrogator (RFID Reader) which transmits and receives the signal and the Transponder (tag) that is attached to the object. RFID leverages electromagnetic or electrostatic coupling in the radio frequency portion of the electromagnetic spectrum to identify objects over a distance of potentially several meters. While its origins can be traced back to 1940s its commercial applications have started expanding significantly recently as a replacement or supplement to barcode technology, thanks in part to standardization, availability of commercial off-the-shelf (COTS) components, and their reducing cost. RFID systems are employed to track shipments and manage supply-chains and to automate toll collection on highways, and are being deployed for many new application areas (e.g., passports, airline boarding passes, luggage tags, etc.). In this work, we experimentally examine the patient details and track them in the case of an emergency in an indoor environment. Our focus is on the people (patients) who do not have any assistance at home particularly the oldies who are totally on bed. Our implementation highlights the monitoring techniques in RFID technology. We have structured our paper in two sections.

\section{BACKGROUND}

With the increasing population it has become important that the details of the patient are maintained in a proper manner. Sometimes it becomes impossible for the patient himself to remember the treatments underwent by him and proper details to be conveyed. According to the recent World Health Organization's (WHO) publication regarding Patient safety, tens of millions i.e. roughly 1.4 million people around the world are injured or dead every year as a result of incorrect medical care. Some of medical errors arise due to:

- Miscommunication,

- Physician order Transcription errors,

- Incomplete Patient Medical Records,

- $\quad$ Overcrowded Situation.

The system proposed here solves all the problems which occurs and is discussed in the objectives mentioned below. 


\subsection{OBJECTIVES}

The proposal in this dissertation focuses on the main objectives as mentioned below:

- To reduce the carrying load of the treatment details and the records.

- To develop a centralized and distributed server and database where the information is shared between different servers

- To provide assistance to patients at home when there is no one beside them.

- To also intimate the relative of the patient and the nearest hospital so that they are there when needed.

\subsection{RFID TAG}

\section{TERMINOLOGIES}

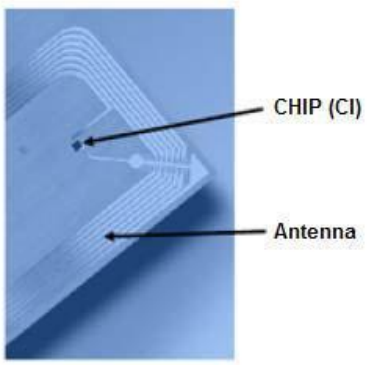

Fig. 1- Tag components

The tags are transponders that have an identifier of the object with which it is associated. The tags typically consist of an antenna and an electronic microchip (Fig 1). The antenna is responsible for making communication between the tag and the reader. There are two main energy classifications of a tag. They can be passive, obtaining energy through the magnetic field generated by readers through antennas, or they can be active, with a battery that provides the energy required to perform processing and modulation of the signal.

\subsection{RFID READER}
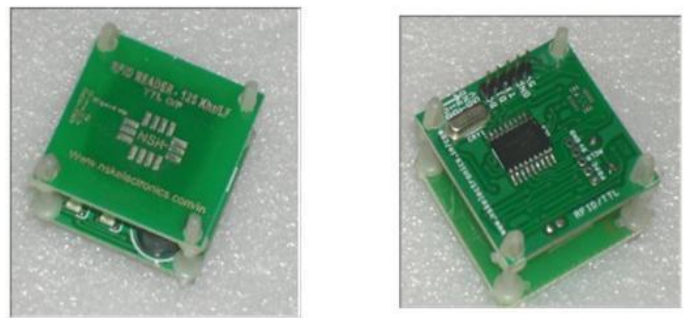

Fig. 2 - RFID Reader with the built-in micro-controller

An RFID Reader (Fig 2) can read through most anything with the exception of conductive materials like water and metal, but with modifications and positioning, even these can be overcome. The RFID Reader emits a low-power radio wave field which is used to power up the tag so as to pass on any information that is contained on the chip. In addition, readers can be fitted with an additional interface that converts the radio waves returned from the tag into a form that can then be passed on to another system, like a computer or any programmable logic controller. Passive tags are generally smaller, lighter and less expensive than those that are active and can be applied to objects in harsh environments, are maintenance free and will last for years.

\subsection{Micro-Controller}

The micro-controller used here is the PIC16F877A. The special features of using this micro-controller are mentioned below:

- 100,000 erase/write cycle Enhanced Flash program memory typical

- 1,000,000 erase/write cycle Data EEPROM memory typical

- Data EEPROM Retention > 40 years

- Self-reprogrammable under software control

- In-Circuit Serial Programming ${ }^{\text {TM }}$ (ICSPTM) via two pins

- Single-supply 5V In-Circuit Serial Programming

- Watchdog Timer (WDT) with its own on-chip RC oscillator for reliable operation

- Programmable code protection

- Power saving Sleep mode

- Selectable oscillator options 
- In-Circuit Debug (ICD) via two pins

\subsection{GSM MODEM}

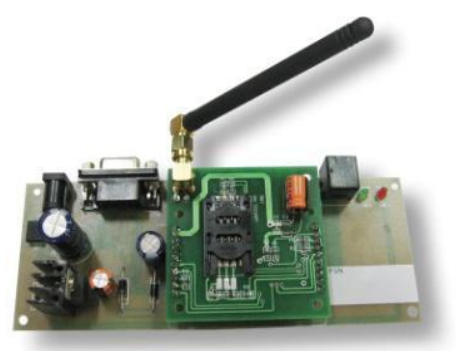

A GSM modem is a specialized type of modem which accepts a SIM card, and operates over a subscription to a mobile operator, just like a mobile phone. From the mobile operator perspective, a GSM modem looks just like a mobile phone. A GSM modem can be a dedicated modem device with a serial, USB or Bluetooth connection, or it may be a mobile phone that provides GSM modem capabilities.

\section{PROPOSED MODEL}

The model here for our project is split here into two parts-

- the monitoring section

- the tracking section.

\subsection{MONITORING SECTION}

\subsubsection{WORKING}

The monitoring section involves the patient being issued the RFID tag if he/she is a new patient; else the reader reads the tag and his/her details like unique ID number, name, age, blood group, treatment details, doctor attended, previous hospital visited etc. at the end the details are updated in the login. In case the patient goes to another hospital then the centralized server shares the information so that the details retrieved from the database are the updated one. So in this way the procedure of carrying the records and the files is totally omitted.

\subsubsection{FLOWCHART}

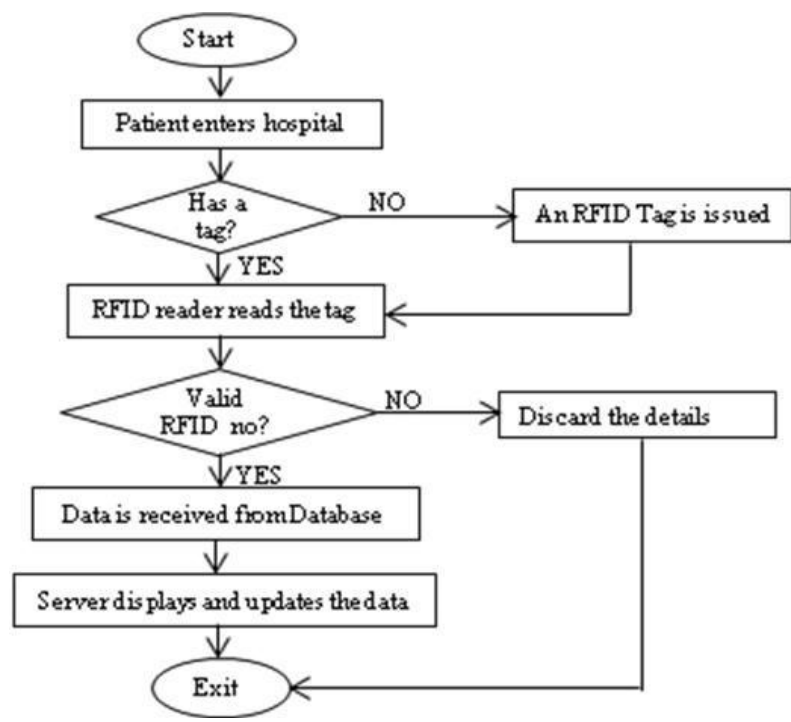

\subsubsection{HARDWARE IMPLEMENTATION}

This hardware is present at the hospital end. The components used here are- RFID Reader where the tag is read whenever a tag is being shown to the reader. A passive tag is used here because it is cost effective.

\subsubsection{EMBEDDED SYSTEM}

The working model which is developed for the monitoring section is displayed as follows with the USB interface attached to the server computer: 


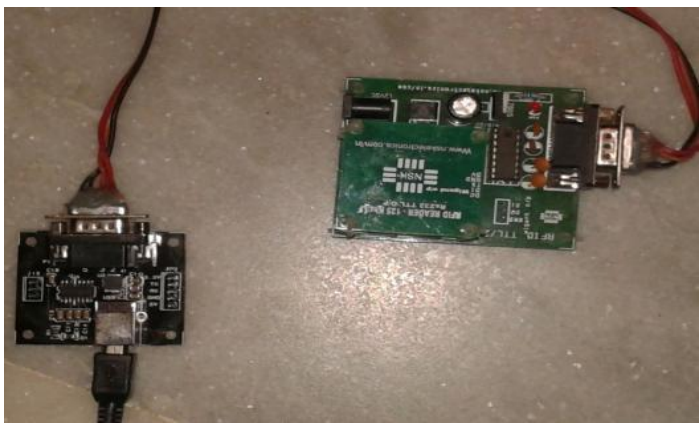

When the RFID tag is shown to the reader, the reader reads the value and is saved in the database. From which it is retrieved by the server and the details are displayed.

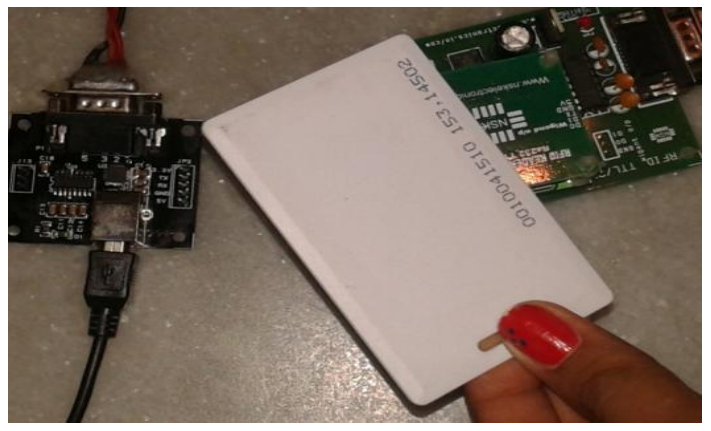

\subsection{Tracking SECTION}

\subsubsection{WORKING}

The tracking section involves the condition and the assistance provided to the patient when he/she is in a closed environment (home) when nobody is around him/her. The patient on the bed is continuously monitored by a temperature and heartbeat sensor. When the value of the temperature rises than the normal body temperature then a SMS is sent to the close relative of the patient along with its location saying the message "TEMPERATURE HIGH". But when the heartbeat raises the normal range then a message is sent to the centralized server hospital with the location. Again if the next minute it remains high then the nearest hospital to the patient's location is calculated and a message is sent to the server of that particular hospital and also a SMS to the relative saying "PATIENT CRITICAL". This way the ambulance reaches the patient through the location received, collects the RFID tag and reaches the hospital for the treatment. When the patient is being taken to the ward the doctor with the RFID tag reads the previous details and treatments undergone and the medications are done accordingly.

\subsubsection{FLOWCHART}

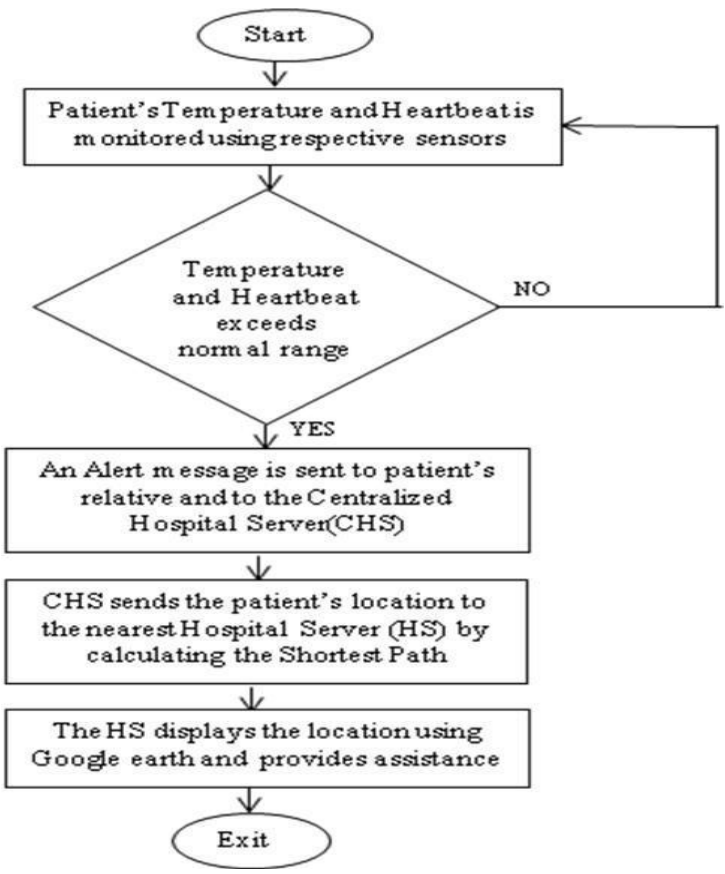




\subsubsection{HARDWARE IMPLEMENTATION}

This hardware is present at the patient's end. The components used here are the GSM Modem, LCD, heartbeat sensor, temperature sensor, PIC16F877A micro-controller and serial to USB interface.

\subsubsection{EMBEDDED SYSTEM}

The working model of the tracking system with both the sensors is displayed below:

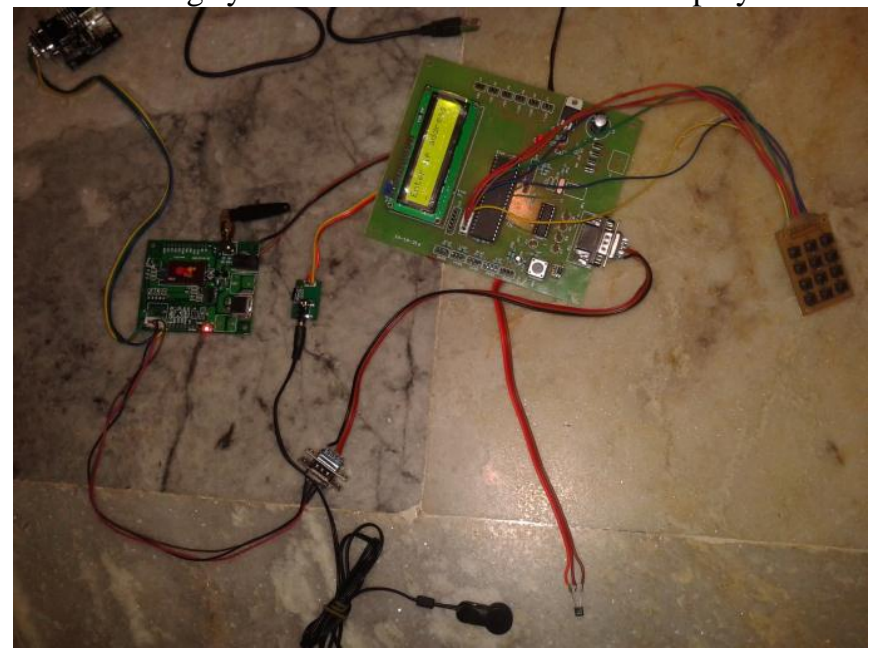

\section{OUTPUT AND RESUlT}

\subsection{MONITORING SECTION IMPLEMENTATION}

\subsubsection{DATABASE MAINTENANCE}

The RFID tag when read refers to the numbers here and submits the details to the server side pages.

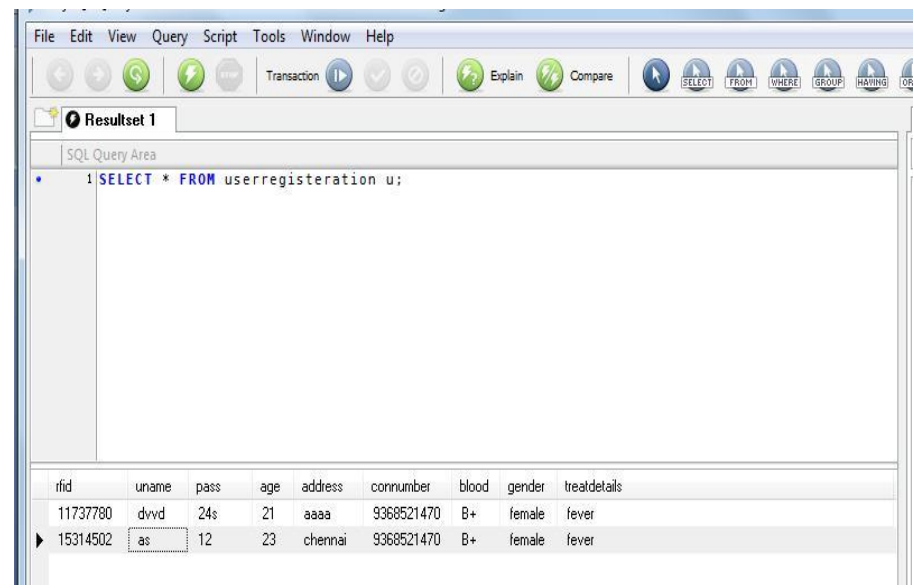

\subsubsection{SERVER SIDE PAgES}

These are those pages which are used for storing and updating the patient's details as and when required.

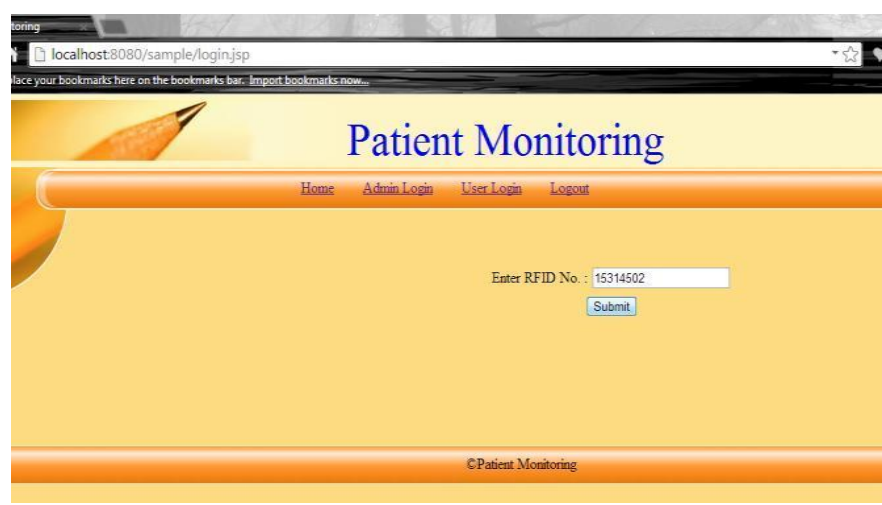




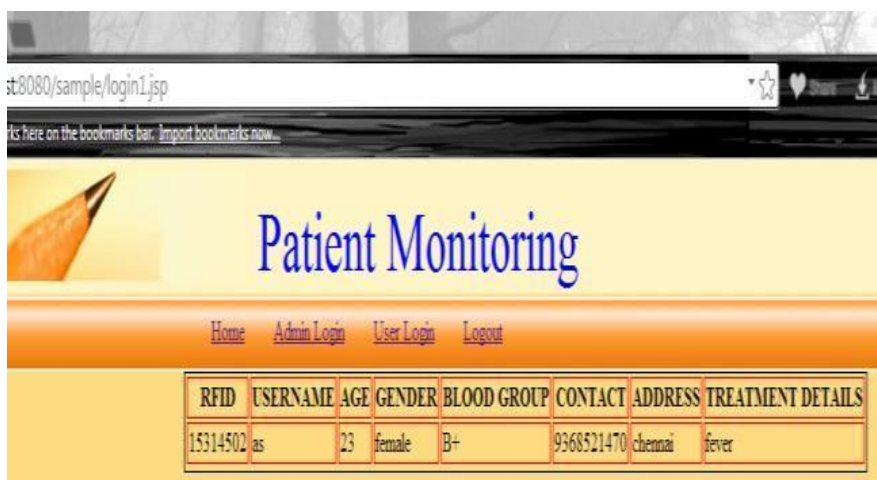

\subsection{TRACKING SECTION IMPLEMENTATION}

\subsubsection{MONITORING OF HEARTBEAT AND TEMPERATURE}

Every minute the heartbeat is recorded and is saved. Only when the second minute the value remains high the message is sent to the Centralized Server Hospital (CHS).

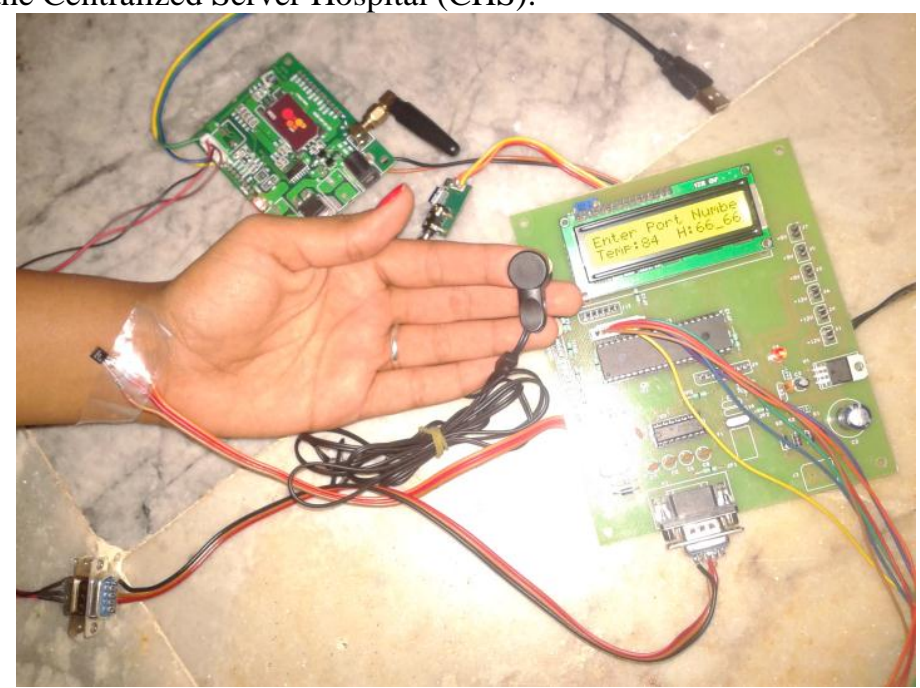

\subsubsection{SMS TO THE RELATIVE}

\subsubsection{Shortest Path Calculation}

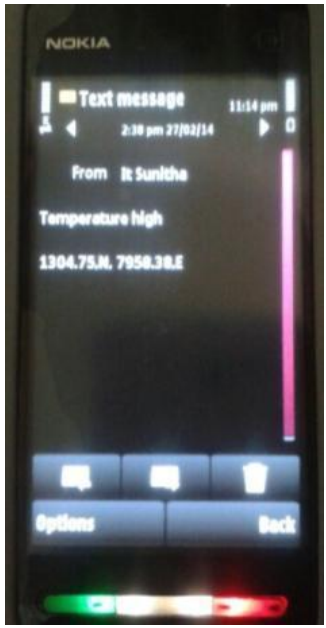

This is calculated at the CHS and arrives at the shortest path with the location. 


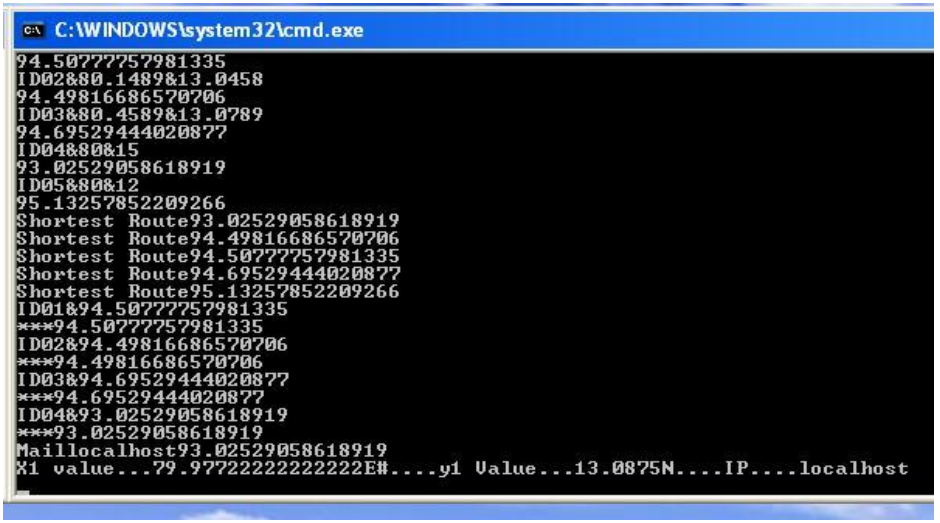

Then the nearest Hospital Server (HS) receives this and provides assistance by sending the ambulance to the patient's location.

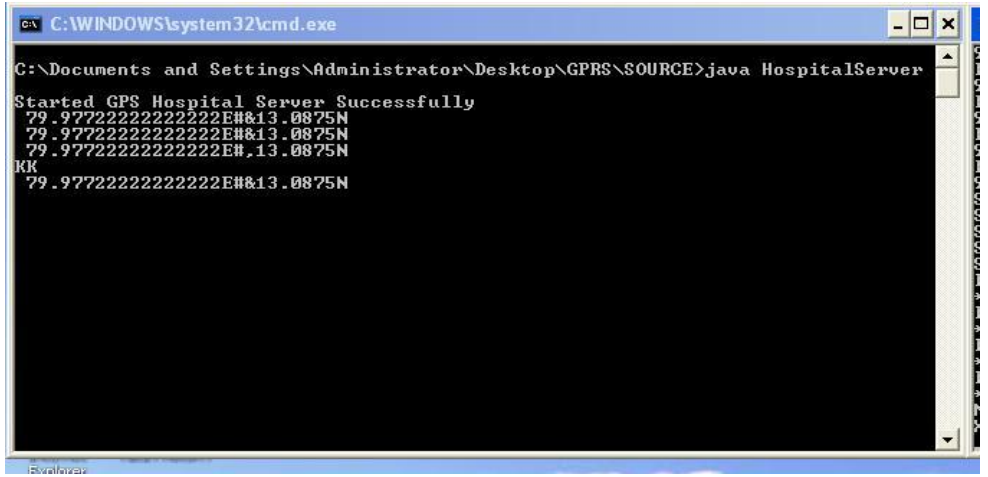

\section{ESTIMATED COST}

The cost of the total project is divided into two parts and then arriving at the total amount.

Monitoring Section -`1500/-

Tracking Section _ - $5500 /$

Total cost of the project _ - $7000 /-$

\section{FUTURE ENHANCEMENT}

In our project we concentrated on the patients who are inside four walls i.e. in a closed environment. In our future work we would develop an application which would be helpful for the people who have got to travel for their work. This would make sure that they are provided assistance on the spot not relying on anyone. Hence reducing the risk of losing their lives in an open environment.

\section{CONCLUSION}

Thus we come to a conclusion of our project where our target was to help the patients who are at home all alone and they have got no one beside them. To provide them assistance in the case of an emergency and to reduce the procedure of carrying the bulk of papers and records during every visit to hospital is our objective.

\section{REFERENCES}

[1] "Reliable and Low Cost RFID Based Authentication System for Large Scale Deployment", "International Journal of Network Security, Vol.14, No.3, PP. 173-179, May 2012"

[2] "RFID enabled smartcards as a context-aware personal health node", "Health Care and Informatics Review Online, 2010, 14(2), pg 10-16, Published online at www.hinz.org.nz

[3] "Cryptanalysis of Security Enhancement for a Modi ed Authenticated Key Agreement Protocol”," International Journal of Network Security, Vol.11, No.1, PP.55\{57, July 2010"

[4] "Privacy and Security Requirements for RFID Applications", "Received 5 April 2008; Revised 21 July 2009; Accepted 25 Aug. 2009 "

[5] "RFID-based Information System for Patients and Medical Staff Identification and Tracking", Tudor Ioan CERLINCA, Cristina TURCU, Cornel TURCU and Marius CERLINCA

[6] "Hospital Automation RFID-Based: Technology Stored In Smart Cards" "Proceedings of the World Congress on Engineering 2008 Vol II WCE 2008, July 2 - 4, 2008, London, U.K."

[7] "Medical Smart Card System for Patient Record Management","Bears Breaking Boundaries 2009 Science, Technology, and Energy Policy White Paper Competition"

[8] "Integrating WSN with Web Services for Patient's Record Management using RFID"," Advance Computing Conference (IACC), 2013 IEEE 3rd International" 
GUIDE: Dr. S. Padmapriya

\section{AUTHOR PROFILE}

Mrs. S. Padmapriya received her B.E. (Electronics and Communication) from Madras University in the year 1991 and M.Tech (Information Technology) from Punjab University and M.E.(Embedded Systems) from Anna University, and PhD (Computer Science) from Berhampur University . She has been the member for evaluation committee for projects and served has Resource coordinator for Bharathdasan University and IGNOU. She has published papers in many national level conferences on embedded systems. She is now presently heading over the Information Technology Department in Prathyusha Institute of Technology and Management.

\section{STUDENTS:}

1. Indu Goel: Pursuing B.Tech (Information Technology)-IV Year in Prathyusha Institute of Technology and Management.

2. A. Sunitha: Pursuing B.Tech (Information Technology)-IV Year in Prathyusha Institute of Technology and Management.

3. P. Arul: Pursuing B.Tech (Information Technology)-IV Year in Prathyusha Institute of Technology and Management. 